\title{
Monte Carlo Simulations of Segregation in Pt-Ni Catalyst Nanoparticles
}

\author{
Guofeng Wang ${ }^{1, *}$, M.A. Van Hove ${ }^{1,2,3}$, P.N. Ross ${ }^{1}$, and M.I. Baskes ${ }^{4}$ \\ ${ }^{1}$ Materials Sciences Division, Lawrence Berkeley National Laboratory, University of \\ California, Berkeley, CA 94720 \\ 2 Advanced Light Source, Lawrence Berkeley National Laboratory, University of \\ California, Berkeley, CA 94720 \\ ${ }^{3}$ Department of Physics, University of California, Davis, CA 95616 \\ ${ }^{4}$ MST-8 Structure and Property Relations Group, Los Alamos National Laboratory, Los \\ Alamos, NM 87545
}

Abstract

We have investigated the segregation of $\mathrm{Pt}$ atoms in the surfaces of Pt-Ni nanoparticles, using Modified Embedded Atom Model potentials and the Monte Carlo method. The nanoparticles are assumed to have disordered fcc configurations at two fixed overall concentrations (50 at.\% Pt and 75 at.\% Pt). We use four kinds of nanoparticle shapes [cube, tetrahedron, octahedron, and cubo-octahedron] terminated by $\{111\}$ and $\{100\}$ facets to examine the extent of the Pt segregation to the nanoparticle surfaces and determine the equilibrium structures of Pt-Ni nanoparticles at $\mathrm{T}=600 \mathrm{~K}$. The model particles contain between 560 and 4631 atoms (particle size ranging from 2.5 to $5 \mathrm{~nm}$ ). Our results imply that a complete $\{100\}$-facet reconstruction could make the cubooctahendral Pt-Ni nanoparticles most energetically favorable, consistent with experimental observations. We predict that at $600 \mathrm{~K}$ due to segregation the equilibrium $\mathrm{Pt}_{50} \mathrm{Ni}_{50}$ nanoparticles with fewer than 2000 atoms and $\mathrm{Pt}_{75} \mathrm{Ni}_{25}$ nanoparticles with fewer than 4000 atoms would achieve a surface-sandwich structure, in which the Pt atoms are enriched in the outermost and third atomic shells while the $\mathrm{Ni}$ atoms are enriched in the second atomic shell. We also find that due to an order-disorder transition the $\mathrm{Pt}_{50} \mathrm{Ni}_{50}$ cubo-octahedral nanoparticles containing more than 2000 atoms would form a core-shell structure with a Pt-enriched surface and a Pt-deficient homogenous core.

*Corresponding author. Fax: (510) 486-5530; Email: gfwang@lbl.gov 


\section{INTRODUCTION}

The surface chemistry of catalyst nanoparticles (also called "clusters") is of great interest because their reactivity and selectivity may be varied by controlling their atomic scale surface structures, e.g. by altering their size and shape [1]. For bimetallic nanoparticles, the geometrical arrangements of the two metals in surfaces are very important for their catalytic behavior [2-4]. However, it is still very difficult to characterize bimetallic nanoparticle surfaces using experimental techniques. Alternatively, theoretical simulations at the atomistic level can provide detailed information and much insight into the surface chemistry of bimetallic nanoparticle systems of interest (see examples in Refs. [5-9]). That is the approach taken in this paper.

The Pt-Ni alloy is an electro-catalyst of interest in the air electrode in low temperature polymer electrolyte fuel cells (PEFCs) [10,11]. It has been reported that the Pt-Ni alloy catalysts may even have enhanced activity compared to pure platinum catalysts, depending on how the surfaces are prepared [11]. Surface segregation phenomena in the Pt-Ni system have been extensively studied both in experiment [12-18] and theory [19-24]. Some very interesting phenomena in the Pt-Ni single crystal alloy surfaces (such as anisotropic surface segregation, oscillatory segregation profiles, and face-related segregation reversal) have been found. However, to our knowledge, there have been no experimental studies of the segregation behavior of Pt-Ni nanoparticles. In

particular, it is of great practical importance to determine if the "buried" subsurface Pt atoms are preferably replaced by $\mathrm{Ni}$ atoms via exchanging with the surface layer in the nanoparticles. Such an exchange would enable us to use less Pt in the electrode yet achieve the same amount of Pt surface atoms (and thus catalytic activity). Distinct from most previous work, we simulate segregation process in both extended surfaces and nanoparticles of Pt-Ni alloys. In this work, we use modified embedded atom model (MEAM) potentials [25] for Pt-Ni alloys and the Monte Carlo simulation method. We chose to develop MEAM potentials rather than using existing EAM potentials [23, 26], because the MEAM leads to better agreement for surface energies of pure metals between theory and experiments than does EAM. 
The paper is structured as follows: in Sec. II we briefly describe the MEAM potentials and Monte Carlo method; in Sec. III we present our calculated results of (111), (100), and (110) surface segregation profiles for Pt-Ni alloys; in Sec. IV we report in detail the equilibrium structures of $\mathrm{Pt}-\mathrm{Ni}$ nanoparticles resulting from surface segregation; finally, the conclusions are drawn in Sec. V.

\section{SIMULATION METHODS}

\section{A. Modified embedded atom method (MEAM)}

Within the MEAM, the total energy of a system is calculated as

$$
E=\sum_{i}\left[F\left(\bar{\rho}_{i}\right)+\frac{1}{2} \sum_{j(\neq i)} \Phi\left(R_{i j}\right)\right]
$$

In the above equation, $\bar{\rho}_{i}$ is the background electron density at the center of atom $\mathrm{i}$ obtained by the superposition of electronic densities from its surrounding atoms. The first term is the embedding energy for atom $i$ which is embedded into the electron density $\bar{\rho}_{i}$, and the second term is the core-core pair repulsion between atom $\mathrm{i}$ and $\mathrm{j}$ separated by a distance $R_{i j}$.

The embedding energy is given as follows:

$$
F(\bar{\rho})=A E_{c}\left(\frac{\bar{\rho}}{\bar{\rho}^{0}}\right) \ln \left(\frac{\bar{\rho}}{\bar{\rho}^{0}}\right)
$$

Here, $A$ is an adjustable parameter, $E_{c}$ is the cohesive energy, and $\bar{\rho}^{0}$ is the density scaling parameter.

The electron density $\bar{\rho}_{i}$ is composed of the spherically symmetric partial electron density $\rho_{i}^{(0)}$ and the angular contributions $\rho_{i}^{(1)}, \rho_{i}^{(2)}$ and $\rho_{i}^{(3)}$. These partial electron densities have the following forms:

$$
\begin{aligned}
& \rho_{i}^{(0)}=\sum_{j \neq i)} \rho_{j}^{a(0)}\left(R_{i j}\right) \\
& \left(\rho_{i}^{(1)}\right)^{2}=\sum_{\alpha}\left[\sum_{j(\neq i)} x_{i j}^{\alpha} \rho_{j}^{a(1)}\left(R_{i j}\right)\right]^{2}
\end{aligned}
$$




$$
\begin{aligned}
& \left(\rho_{i}^{(2)}\right)^{2}=\sum_{\alpha, \beta}\left[\sum_{j \neq i)} x_{i j}^{\alpha} x_{i j}^{\beta} \rho_{j}^{a(2)}\left(R_{i j}\right)\right]^{2}-\frac{1}{3}\left[\sum_{j(\neq i)} \rho_{j}^{a(2)}\left(R_{i j}\right)\right]^{2} \\
& \left(\rho_{i}^{(3)}\right)^{2}=\sum_{\alpha, \beta, \gamma}\left[\sum_{j \neq i} x_{i j}^{\alpha} x_{i j}^{\beta} x_{i j}^{\gamma} \rho_{j}^{a(3)}\left(R_{i j}\right)\right]^{2}-\frac{3}{5} \sum_{\alpha}\left[\sum_{j \neq i} x_{i j}^{\alpha} \rho_{j}^{a(3)}\left(R_{i j}\right)\right]^{2}
\end{aligned}
$$

Here, $x_{i j}^{\alpha}=R_{i j}^{\alpha} / R_{i j}$, and $R_{i j}^{\alpha}$ is the $\alpha$ component $(\alpha=\mathrm{x}, \mathrm{y}$, or $\mathrm{z})$ of the distance vector between atom i and j. $\rho_{j}^{a(h)}\left(R_{i j}\right)=e^{-\beta^{(h)}\left(R_{i j} / r_{e}-1\right)}(\mathrm{h}=0,1,2$, and 3) represents the atomic electron densities contributed from atom $j$ to atom i, $\beta^{(\text {h) }}$ are adjustable parameters, and $r_{e}$ is the nearest-neighbor distance in the equilibrium reference structure. Note that Eq. (3d) is different from that in earlier work [25]. This new modification makes the partial electron densities orthogonal [27]. In this work, we use the following scheme (see other schemes in Ref. 28) to combine the partial electron densities and compute the background electron density:

$$
\begin{aligned}
\bar{\rho}_{i} & =\rho_{i}^{(0)} \sqrt{1+\Gamma_{i}} \\
\Gamma_{i} & =\sum_{h=1}^{3} t^{(h)}\left[\rho_{i}^{(h)} / \rho_{i}^{(0)}\right]^{2}
\end{aligned}
$$

where $\mathrm{t}^{(\mathrm{h})}$ are adjustable parameters.

The pair potential between two atoms separated by a distance $\mathrm{R}$ is given by:

$$
\Phi(R)=\frac{2}{Z}\left[E^{u}(R)-F\left(\bar{\rho}^{0}(R)\right)\right]
$$

where $\mathrm{Z}$ is the number of nearest neighbors in the bulk reference structure ( $\mathrm{Z}=12$ for fcc metals). $E^{u}(R)$ is the energy per atom of the reference structure as a function of nearestneighbor distance $\mathrm{R}$ and is determined using the following universal equation of state of Rose et al. [29].

$$
\begin{aligned}
& E^{u}(R)=-E_{c}\left(1+a^{*}\right) e^{-a *} \\
& a^{*}=\alpha\left(R / r_{e}-1\right) \\
& \alpha=\sqrt{\frac{9 \Omega B}{E_{c}}}
\end{aligned}
$$


Here, $\mathrm{E}_{\mathrm{c}}, \mathrm{r}_{\mathrm{e}}, \Omega$, and $\mathrm{B}$ are the cohesive energy, nearest-neighbor distance, atomic volume, and bulk modulus for the equilibrium reference structure, respectively.

$\bar{\rho}^{0}(R)$ in Eq. (5) is the background electron density for the reference structure.

For fcc metals,

$$
\bar{\rho}^{0}(R)=Z \rho^{a(0)}(R)
$$

\section{B. Screening procedure}

The current version of the MEAM considers only nearest-neighbor interactions, therefore, we must provide a screening procedure to define which are the nearest neighbors of an atom. We use the many-body screening function proposed by Baskes [28] using the elliptical construction.

The screening function $\mathrm{S}_{\mathrm{ik}}$ between atom $\mathrm{i}$ and atom $\mathrm{k}$ depends on all the other atoms between them, thus

$$
\begin{aligned}
& S_{i k}=\prod_{j \neq i, k} S_{i j k} \\
& S_{i j k}=f_{c}\left(\frac{C-C_{\text {min }}}{C_{\text {max }}-C_{\text {min }}}\right)
\end{aligned}
$$

where $f_{c}(x)$ is a function of the material dependent parameters $\mathrm{C}_{\max }$ and $\mathrm{C}_{\min }$. The parameter $\mathrm{C}$ is determined using the following equation.

$$
C=\frac{2\left(X_{i j}+X_{j k}\right)-\left(X_{i j}-X_{j k}\right)^{2}-1}{1-\left(X_{i j}-X_{j k}\right)^{2}}
$$

where $X_{i j}=\left(r_{i j} / r_{i k}\right)^{2}$ and $X_{j k}=\left(r_{j k} / r_{i k}\right)^{2}$. The $\mathrm{r}_{\mathrm{ij}}, \mathrm{r}_{\mathrm{jk}}$, and $\mathrm{r}_{\mathrm{ik}}$ are the distances between the corresponding atoms.

The smooth cutoff function $\mathrm{f}_{\mathrm{c}}(\mathrm{x})$ in Eq. (8b) assumes the following form.

$$
\begin{aligned}
& \text { If } x \geq 1, \quad f_{c}(x)=1 \\
& \text { If } 0<x<1, f_{c}(x)=\left[1-(1-x)^{4}\right]^{2} \\
& \text { If } x \leq 0, \quad f_{c}(x)=0
\end{aligned}
$$


We multiply the atomic electron density and the pair potentials by the screening function $S_{i k}$, hence $S_{i k}=1$ represents unscreened interaction while $S_{i k}=0$ represents a completely screened interaction.

\section{Monte Carlo method}

The employed Monte Carlo (MC) simulation method is based on the Metropolis algorithm [30]. The MC method is particularly advantageous in studying segregation phenomena in the equilibrium alloy structure, because they can circumvent slow physical dynamic processes (such as diffusion) in the system and provide an averaged composition profile over a thermodynamic equilibrium ensemble [31].

In our calculations, we used statistical mechanics with a canonical ensemble, where the total number of atoms of each element and the temperature are constants. In this approach, starting from some atomic configuration, the series of configurations are generated in proportion to the probabilities of a configuration occurring in the equilibrium ensemble. In each step, one of the following two configuration transformations is tried out with an equal probability:

(1) A randomly selected atom is displaced from its original position in a random direction. The magnitude of the displacement is in the range of $\left(0, r_{\max }\right]$. At a given temperature, the maximum displacement $r_{\max }$ is tuned so that the acceptance rate of new configurations is about 0.5 during the equilibrated part of the simulations.

(2) Two randomly selected atoms with different element types are exchanged.

The operation (1) accounts for the relaxation and vibration processes, and the operation (2) accounts for the inter-diffusion process in the model system.

According to the Boltzmann distribution, the probability $\left(\mathrm{P}_{\mathrm{XY}}\right)$ of the configuration transformation (from $\mathrm{X}$ to $\mathrm{Y}$ ) is given by a Boltzmann factor for the change in energy $\left(\Delta \mathrm{E}=\mathrm{E}_{\mathrm{Y}}-\mathrm{E}_{\mathrm{X}}\right)$.

$$
P_{X Y}=\exp \left(-\frac{\Delta E}{k_{B} T}\right)
$$


Here, $\mathrm{k}_{\mathrm{B}}$ is Boltzmann constant and $\mathrm{T}$ is the temperature. If $\mathrm{P}_{\mathrm{XY}} \geq 1$ (decrease in energy), the new configuration is always retained, while if $\mathrm{P}_{\mathrm{XY}}<1$ (increase in energy), the new configuration is retained with the probability $\mathrm{P}_{\mathrm{XY}}$.

After repeating the above procedures for multi-million MC steps, the physical quantities such as the composition profile are obtained by averaging over the resulting configurations. For example, we used the above Monte Carlo scheme to determine the lattice constants for disordered fcc $\mathrm{Pt}_{75} \mathrm{Ni}_{25}$ and $\mathrm{Pt}_{50} \mathrm{Ni}_{50}$ bulk alloys. To this end, we carried out $\mathrm{MC}$ simulations for disordered fcc $\mathrm{Pt}_{75} \mathrm{Ni}_{25}$ and $\mathrm{Pt}_{50} \mathrm{Ni}_{50}$ bulk alloys with various lattice parameters and determined the lattice constant that led to the zero average pressure (strain) for a cubic 3D periodic simulation cell containing 500 atoms at a given temperature. The whole MC simulation takes 5 million steps, but we only average the pressure for simulation cells for the last 3 million steps to eliminate the influence from the original structure. For the $\mathrm{Pt}_{75} \mathrm{Ni}_{25}$ bulk alloy the equilibrium lattice constants are found to be $\mathrm{a}=3.842 \AA$ at $600 \mathrm{~K}$ and $\mathrm{a}=3.862 \AA$ at $1200 \mathrm{~K}$. For the $\mathrm{Pt}_{50} \mathrm{Ni}_{50}$ bulk alloy the equilibrium lattice constants are $a=3.743 \AA$ at $600 \mathrm{~K}$ and $\mathrm{a}=3.768 \AA$ at $1200 \mathrm{~K}$. The calculated lattice constants reasonably agree with $\mathrm{a}=3.836 \AA$ for the $\mathrm{Pt}_{75} \mathrm{Ni}_{25}$ bulk alloy and $\mathrm{a}=3.749 \AA$ for the $\mathrm{Pt}_{50} \mathrm{Ni}_{50}$ bulk alloy interpolated from experimental data [32]. These lattice constants are used to calculate the initial atomic separations in the surface (Sec. III.B) and nanoparticle (Sec. IV) simulations. In this work, we chose to simulate segregation in extended surfaces of disordered Pt-Ni alloys at $\mathrm{T}=1200 \mathrm{~K}$ for a direct comparison with previous experiments [12-18] and simulations [19-24]. $\mathrm{T}=600 \mathrm{~K}$ is a typical reduction temperature for bimetallic nanoparticle catalysts [5] and is used here to investigate segregation in Pt-Ni nanoparticles.

\section{MEAM DESCRIPTION OF Pt-Ni}

\section{A. Development of the potentials}

The MEAM potentials for pure $\mathrm{Pt}$ and pure $\mathrm{Ni}$ are the same potentials published before $[25,28]$ except for the renormalization of parameters $t^{(h)}$ caused by the modification in Eq. 3(d). To determine the cross potentials between Pt and Ni, we chose 
$\mathrm{Pt}_{3} \mathrm{Ni}$, which adopts the $\mathrm{L}_{2}$ structure, as the reference structure. Hence, the Pt-Ni pair potential is evaluated using the following expression [33].

$$
\Phi_{P t N i}(R)=\frac{1}{3} E_{P t_{3} N i}^{u}(R)-\frac{1}{4} F_{P t}\left(\bar{\rho}_{P t}\right)-\frac{1}{12} F_{N i}\left(\bar{\rho}_{N i}\right)-\Phi_{P t P t}(R)
$$

where

$$
\begin{aligned}
& \bar{\rho}_{P t}=\sqrt{\left[8 \rho_{P t}^{a(0)}(R)+4 \rho_{N i}^{a(0)}(R)\right]^{2}+\frac{8}{3} t_{P t}^{(2)}\left[\rho_{P t}^{a(2)}(R)-\rho_{N i}^{a(2)}(R)\right]^{2}} \\
& \bar{\rho}_{N i}=12 \rho_{P t}^{a(0)}(R)
\end{aligned}
$$

The parameters for the Pt-Ni MEAM potentials are given in Table I, and Table II gives the angular screening parameters for the potentials. We also in Table III give the calculated surface energies of the relaxed low index surfaces for pure Pt and pure $\mathrm{Ni}$ using our MEAM potentials. The calculated surface energies using the MEAM potentials agree quite well with the $a b$ initio calculations [34][density-functional theory (DFT) with the generalized gradient approximation (GGA)] and experiments $[35,36]$. It is noticeable that the surface energies in Table III for pure Pt and pure Ni are pretty close. Therefore, the surface energy difference is not a major factor determining surface segregation behavior for Pt-Ni alloys.

We fit the MEAM cross potentials between $\mathrm{Pt}$ and $\mathrm{Ni}$ with first-principles calculation results of (1) the three elastic constants for $\mathrm{Pt}_{3} \mathrm{Ni}$ (L12) and (2) the heat of formation for $\mathrm{Pt}_{3} \mathrm{Ni}\left(\mathrm{L}_{2}\right)$, and check the transferability of our MEAM using firstprinciples calculation results of the heat of formation of $\mathrm{PtNi}_{3}\left(\mathrm{~L}_{2}\right)$, and $\mathrm{PtNi}\left(\mathrm{L}_{1}\right)$. Table IV shows a good agreement between our MEAM results and first-principles calculations for properties of bulk Pt-Ni intermetallic compounds. To obtain the above first principles calculation results, we evaluated the system energies using densityfunctional theory with the local spin density approximation (LSDA). We employed the PARATEC code [37], a massively parallel package performing ab initio quantummechanical total energy calculations using pseudopotentials and a plane wave basis set. In this work, we used the package FHI98PP [38] to generate the norm-conserving Troullier-Martins [39] type of pseudopotentials, employing common parametrizations of the local-density approximation for exchange and correlation. In all calculations, we have used a 16x16x16 k-point grid for k-space integration and a kinetic energy cutoff of $90 \mathrm{Ry}$ 
to expand the electronic wave-functions in the plane wave base. The elastic constants are determined following the procedure given in Ref. [40].

In the dilute limit, the segregation energy of extended surfaces is just the energy difference in the total energy of the system with a substitutional impurity atom in a surface layer and in the bulk. A negative value of this energy implies that the impurity will be enriched in that surface layer. A positive value thus implies deletion of that element. The calculated segregation energies are considered as useful material properties indicating the segregation species and suggesting the magnitude of the segregation in surfaces. Therefore, we tuned up the parameters of the Pt-Ni MEAM potentials leading to the segregation energies in Table V for (111), (100), and (110) surfaces. These segregation energies for extended surfaces qualitatively predict surface segregation phenomena agreeing with experimental results.

The segregation energy is $0.27 \mathrm{eV}$ for $\mathrm{Ni}$ in the first layer of $\mathrm{Pt}$ (111) surfaces and $-0.35 \mathrm{eV}$ for Pt in the first layer of $\mathrm{Ni}$ (111) surfaces. Hence, it is anticipated that $\mathrm{Pt}$ atoms will be enriched in the first layer of (111) surfaces of Pt-Ni alloys. Actually, our results from Monte Carlo simulations in the next subsection confirm this prediction. Moreover, the calculated segregation energies using our MEAM potentials are comparable to the ab initio results [41] (0.43 eV for $\mathrm{Ni}$ in $\mathrm{Pt}(111)$ and $-0.17 \mathrm{eV}$ for $\mathrm{Pt}$ in $\mathrm{Ni}$ (111)). In the second layer of (111) surfaces, Ni in Pt surfaces lead to negative segregation energy and $\mathrm{Pt}$ in $\mathrm{Ni}$ surfaces lead to positive segregation energy. Thus, Ni will be enriched in the second layer of (111) surfaces of Pt-Ni alloys. These predictions of segregation phenomena for (111) surfaces of Pt-Ni alloys using the MEAM segregation energies qualitatively agree with the experimental measurements [12, 42-44].

For (100) surfaces, the segregation energies for both $\mathrm{Ni}$ in $\mathrm{Pt}(100)$ and $\mathrm{Pt}$ in $\mathrm{Ni}$ (100) are negative. However, Pt in the first layer of Ni (100) surfaces leads to a more negative segregation energy than its counterpart. Thus, the Pt atoms will be enriched in the first layer of (100) surfaces. Ni in the second layer of Pt (100) surfaces leads to a more negative segregation energy than vice versa and hence the $\mathrm{Ni}$ atoms will be enriched in the second layer of (100) surfaces of Pt-Ni alloys. Using previous EAM potentials [26], the segregation energies for $\mathrm{Ni}$ in $\mathrm{Pt}$ (100) surfaces are determined to be: - 
$0.01 \mathrm{eV}$ in the first layer and $-0.20 \mathrm{eV}$ in the second layer; the segregation energies for $\mathrm{Pt}$ in $\mathrm{Ni}(100)$ surfaces are: $-0.32 \mathrm{eV}$ in the first layer and $0.00 \mathrm{eV}$ in the second layer. These EAM potentials give an accurate description of segregation profile in (100) surfaces of Pt-Ni alloys $[19,21]$. Our MEAM results of segregation energy in Table V agree well with the EAM calculations.

It is an unusual phenomenon that $\mathrm{Ni}$ atoms (rather than Pt atoms with large size) will strongly segregate to the first layer of (110) surfaces of Pt-Ni alloys. First-principles calculations [22] attribute this segregation reversal to the combination of a strong segregation of Pt atoms to the second layer of (110) surfaces and a strong tendency of forming Pt-Ni nearest-neighboring pairs in Pt-Ni alloys. Our results of segregation energies from MEAM potentials suggest a similar physical picture. As shown in Table V, the segregation energies for Pt in both the first and second layers of $\mathrm{Ni}$ (110) surfaces are negative. However, the segregation of Pt atoms to the second layer is more energetically favorable and thus the Pt atoms should be enriched in the layer of (110) surfaces. The negative cohesive energies for $\mathrm{Pt}_{3} \mathrm{Ni}\left(\mathrm{L}_{2}\right), \mathrm{PtNi}_{3}\left(\mathrm{~L}_{2}\right)$, and $\mathrm{PtNi}\left(\mathrm{L}_{0}\right)$ in Table IV indicate it is more energetically favorable to form a Pt-Ni nearest-neighboring pair than $\mathrm{Pt}-\mathrm{Pt}$ and Ni-Ni in Pt-Ni alloys. Therefore, it is expected that the concentration of $\mathrm{Pt}$ atoms in the first layer of (110) surfaces will be lower, i.e. Ni atoms segregate to the first layer of (110) surfaces of Pt-Ni alloys.

\section{B. Monte Carlo simulation of segregation in extended surfaces}

In order to illustrate the applicability of our MEAM potentials to studying segregation phenomena in Pt-Ni alloys, we further employed MC simulations to predict segregation profiles for three low-index extended Pt-Ni alloy surfaces: (111), (100), and (110). To simulate extended surfaces, we used slab simulation cells in which periodic boundary conditions are applied in the two directions parallel to the surface. There are two surfaces in each slab simulation cell. The number of surface atoms, which are only the atoms in the outermost layer on one side of the slab, was 36 for (111) surfaces, 32 for (100) surfaces, and 24 for (110) surfaces. There are 15 layers for (111) surfaces, 17 layers for (100) surfaces, and 24 layers for (110) surfaces in our slab simulation cells. Thus, the bulk surfaces were simulated using a 2D periodic slab containing 540 atoms for (111) 
surfaces, 544 atoms for (100) surfaces, and 576 atoms for (110) surfaces. The initial simulation cells assume fcc structures with the lattice constant determined for the bulk alloy at temperature $\mathrm{T}=1200 \mathrm{~K}$ and the composition 50 at. $\%\left(\mathrm{Pt}_{50} \mathrm{Ni}_{50}\right)$ or 75 at.\% $\left(\mathrm{Pt}_{75} \mathrm{Ni}_{25}\right)$ of Pt. Thus, the simulation slabs are about $30 \AA$ thick.

For comparison, there are results from low energy electron diffraction (LEED) [15] and first-principles calculations [22] for segregation profiles of the three low-index extended surfaces in disordered $\mathrm{Pt}_{50} \mathrm{Ni}_{50}$ alloys. Therefore, we first calculated those surface segregation profiles. Moreover, we will show the significant effect of surface relaxations on surface segregation processes. To this end, we devised the following MC simulation with different schemes to simulate various relaxation processes.

Scheme 1: No relaxation. The atoms in the simulation slab are in their bulkterminated positions and their positions are fixed during the MC simulations.

Scheme 2: Interlayer relaxation from LEED measurements. The atoms in the simulation slab are in their bulk-terminated positions but with interlayer relaxation determined by LEED [15]; the positions of atoms are fixed during the MC simulations.

Scheme 3: Interlayer relaxation from MC simulations. The atoms are initially in their bulk-terminated positions but allowed to move in the direction normal to the surface by a random displacement within $(0,0.02 \AA]$.

Scheme 4: Full relaxation from MC simulations. The same procedure described in Sec. II.C: atoms can move in any direction.

In all $\mathrm{MC}$ simulations, the $\mathrm{Pt}$ and $\mathrm{Ni}$ atoms were initially distributed randomly in the whole simulation slab and the atoms with different type of elements are allowed to exchange their element types based on Eq. (11). For each combination of scheme and surface orientation, we carried out MC simulation for 8 million steps at $\mathrm{T}=1200 \mathrm{~K}$. To eliminate the influence of the initial configurations, we discarded the first 3 million MC steps and sampled the composition profile every 1000 steps in the last 5 million MC steps.

We report in Table VI our calculated segregation profiles for extended surfaces of disordered $\mathrm{Pt}_{50} \mathrm{Ni}_{50}$ alloys from schemes 1, 2, and 3. It can be seen that our calculations using the MEAM potentials reproduce the previously observed major features of 
segregation phenomena in $\mathrm{Pt}_{50} \mathrm{Ni}_{50}$ extended surfaces: oscillatory segregation profiles and (110) surface segregation reversal. Our results show that (1) in (111) and (100) surfaces $\mathrm{Pt}$ is enriched in the first and third layers, while $\mathrm{Ni}$ is enriched in the second layer; in (110) surfaces $\mathrm{Ni}$ is enriched in the first and third layers while $\mathrm{Pt}$ is enriched in the second layer. Furthermore, our calculated segregation profiles using scheme 2 and 3 agree excellently with the LEED measurements and first-principles calculations. In contrast, the calculated segregation profiles using scheme 1 without interlayer relaxations are much different, especially for (110) surfaces. These results indicate that interlayer relaxation is an important factor in determining surface segregation profiles in Pt-Ni alloys.

Now, we discuss our surface segregation results, which are not included in Table $\mathrm{VI}$, using $\mathrm{MC}$ simulations with scheme 4 for $\mathrm{Pt}_{50} \mathrm{Ni}_{50}$ alloys. These simulations for the (111) surface lead to similar segregation profile as using the other schemes. In contrast, surface reconstruction occurs during the simulations for both (100) and (110) surfaces. In the outermost layer of (100) surfaces, the initial square lattice determined by bulk termination tends to reconstruct to a denser hexagonal lattice after many MC steps. This finding is consistent with previous experimental observations for the (100) surface of PtNi alloys [17,18]. Particularly, it was found in a LEED study [17] that the top layer of the $\mathrm{Pt}_{50} \mathrm{Ni}_{50}(100)$ surface reconstructs to $(12 \times 1)$ and $(19 \times 1)$ superstructures that are quasihexagonal atomic meshes with almost (111) atomic density. Our MC simulations indicate the same reconstruction process and predict Pt enrichment in the outermost layer of the (100) surface, agreeing with experiments [17]. Our simulations using scheme 4 also found a surface reconstruction process for (110) surfaces. In contrast, a LEED study [13] shows that a large fraction of their $\mathrm{Pt}_{50} \mathrm{Ni}_{50}$ (110) surface sample is not reconstructed and only some small patches correspond to reconstructed (1x2) and (2x1) domains. It appears that our MEAM potentials overestimate surface reconstruction tendency for (110) surfaces of Pt-Ni alloys. This should not be a concern in our following work for Pt-Ni nanoparticles, because (110) surfaces are less stable in fcc crystals and do not appear as a facet of nanoparticles. It is worth emphasizing that our MC simulations using scheme 4 can not simulate the actual reconstructed (100) and (110) surfaces accurately. To do that would require a much larger simulation cell and an $\mathrm{MC}$ algorithm that allows changes in 
the number of atoms from the fcc-crystal terminated surface configurations. It is also worth pointing out that none of previous theoretical work [19-24] explicitly takes the correct surface reconstruction into account when determining surface segregation profiles of Pt-Ni alloys.

Table VII gives the calculated segregation profiles for $\mathrm{Pt}_{75} \mathrm{Ni}_{25}$ surfaces, showing the oscillatory segregation profiles and the (110) surface segregation reversal. Our results for the (111) surface are close to the measured Pt concentration of 99 at.\%, 30 at. \%, and 87 at.\% in the first, second, and third layers of the $\mathrm{Pt}_{78} \mathrm{Ni}_{22}$ (111) surface [12]. Since the (111) surface appears most frequently in fcc polycrystalline surfaces due to its low energy, our finding (97 at.\% of $\mathrm{Pt}$ atoms in the outermost layer of $\mathrm{Pt}_{75} \mathrm{Ni}_{25}$ (111) surfaces) also agrees with the low-energy ion-scattering (LEIS) spectroscopy results [10]. It is noticed that a (110) surface segregation reversal for $\mathrm{Pt}_{75} \mathrm{Ni}_{25}$ alloys is predicted only after permitting interlayer relaxation. Not surprisingly, we also find the surface reconstruction in (100) and (110) surfaces of $\mathrm{Pt}_{75} \mathrm{Ni}_{25}$ alloys using the $\mathrm{MC}$ simulations with full relaxation (scheme 4).

In summary, we have developed MEAM potentials for Pt-Ni alloys that are capable of predicting surface segregation and surface reconstruction consistent with experimental and first-principles results.

\section{SEGREGATION IN Pt-Ni NANOPARTICLES}

To investigate the segregation of $\mathrm{Pt}$ atoms to surfaces and determine the equilibrium shape, we simulated Pt-Ni nanoparticles with four different shapes: cube (Fig. 1(a)), tetrahedron (Fig. 1(b)), octahedron (Fig. 1(c)), and cubo-octahedron (Fig. 1(d)). They all initially have the fcc crystal structure. To study the size effect, we chose sequences of "magic" numbers of atoms (i.e., nanoparticles containing complete shells of atoms) for each kind of nanoparticles to be about 600, 1000, 2000, and 4000. Our work is stimulated by the report that the shapes and sizes of (at least platinum) nanoparticles could be controlled in the synthesis process [45].

In bulk fcc materials, each atom has twelve nearest neighbors (nn) as shown in Fig. 1(e). In contrast, atoms on the extended surface or nanoparticles of fcc alloys have an 
incomplete set of nearest neighbors. For example, atoms on $\{100\}$ surfaces have only eight nearest neighbors (8nn) and the atoms 1, 2, 4, and 5 (numbered as in Fig. 1(e)) are missing, while atoms on $\{111\}$ surfaces have nine nearest neighbors $(9 \mathrm{nn})$ and the atoms 1, 2, and 3 (numbered as in Fig. 1(e)) are absent. Following the approach proposed in Ref. [46] based on the number and arrangement of nearest neighboring atoms, we can distinguish atoms at various sites (facet, edge and vertex) on the surface of nanoparticles.

- In a cubic nanoparticle (Fig. 1(a)), there are six $\{100\}$ facets (8nn), twelve $\{100\} /\{100\}$ edges (5nn), and eight vertices (3nn).

- In a tetrahedral nanoparticle (Fig. 1(b)), there are four $\{111\}$ facets (9nn), six $\{111\} /\{111\}$ edges (6nn), and four vertices (3nn).

- In an octahedral nanoparticle (Fig. 1(c)), there are eight $\{111\}$ facets (9nn), twelve $\{111\} /\{111\}$ edges (6nn), and six vertices (3nn).

- In a cubo-octahedral nanoparticle (Fig. 1(d)), there are six $\{100\}$ facets (8nn), eight $\{111\}$ facets (9nn), twelve $\{111\} /\{111\}$ edges (7nn), twenty four $\{111\} /\{100\}$ edges $(7 \mathrm{nn})$, and twenty four vertices (6nn).

In what follows, we present our simulation results of segregation in un-relaxed (Sec. IV.A) and relaxed (Sec. IV.B) Pt-Ni nanoparticles.

\section{A. Segregation in un-relaxed Pt-Ni nanoparticles}

To find the shapes that are most close to equilibrium nanoparticle configurations, we first performed the Monte Carlo simulations for Pt-Ni nanoparticles with the four different shapes neglecting atomic displacements. In this way, we can eliminate those nanoparticle shapes that are only meta-stable before the extensive MC simulations considering atomic relaxation. The separations of atoms in the nanoparticles are determined by the lattice constants of the bulk alloys at the same composition and a temperature of $600 \mathrm{~K}$ (see details in Sec. II.C). The Pt and Ni atoms were initially distributed randomly in the nanoparticles. For each nanoparticle, we carried out the MC simulation for 10 million $\mathrm{MC}$ steps at $\mathrm{T}=600 \mathrm{~K}$. To eliminate the influence of the initial configurations, we discarded the first 2 million MC steps and sampled the physical quantities every 1000 steps in the last 8 million MC steps. 
The atomic cohesive energy, which is the total potential energy (U) divided by the number of atoms $(\mathrm{N})$, is a parameter indicating the relative stability of nanoparticles with different shapes. Figures 2(a) and 2(b) show our calculated atomic cohesive energy as a function of the number of atoms for four kinds of nanoparticles. For every kind of nanoparticle (distinguished by shape and composition) in our study, an approximately linear relation between $\mathrm{U} / \mathrm{N}$ and $\mathrm{N}^{-1 / 3}$ is observed.

$$
\frac{U}{N} \approx E_{B}+k \cdot N^{-\frac{1}{3}}
$$

In the above equation, $\mathrm{E}_{\mathrm{B}}(<0)$ is the atomic cohesive energy for atoms in bulk materials. The term $\mathrm{k} \cdot \mathrm{N}^{-1 / 3}$ represents the contribution to the cohesive energy from surface atoms, whose number is approximately proportional to $\mathrm{N}^{2 / 3}$. A similar relation of atomic cohesive energy and number of atoms was found previously for pure metal nanoparticles $[47,48]$.

In both $\mathrm{Pt}_{50} \mathrm{Ni}_{50}$ (Fig. 2(a)) and $\mathrm{Pt}_{75} \mathrm{Ni}_{25}$ (Fig. 2(b)) fcc nanoparticles, the atomic cohesive energy for a given number of atoms follows the decreasing order: cube $>$ tetrahedron $>$ octahedron $\approx$ cubo-octahedron. For $\mathrm{Pt}_{50} \mathrm{Ni}_{50}$ nanoparticles, the cubooctahedral shape is about $0.003 \mathrm{eV} /$ atom more stable than the octahedral shape. In contrast, the average energy for each atom in the $\mathrm{Pt}_{75} \mathrm{Ni}_{25}$ octahedral nanoparticles is about $0.001 \mathrm{eV}$ lower than in the cubo-octahedral nanoparticles. It is noticeable that the decreasing order of atomic energy for the four shapes of nanoparticles does not always correlate with the decreasing order of their surface/volume ratios, which is tetrahedron > cube $>$ octahedron $>$ cubo-octahedron. This is because $\{100\}$ facets in nanoparticles have higher surface energies than $\{111\}$ facets with the same area. Table III shows that our calculated energy of extended (100) surfaces is higher by $504 \mathrm{~mJ} / \mathrm{m}^{2}$ for elemental Pt and by $399 \mathrm{~mJ} / \mathrm{m}^{2}$ for elemental Ni compared to extended (111) surfaces. Therefore, the cubic nanoparticles terminated with $\{100\}$ facets will have higher energies than the tetrahedral nanoparticles terminated with $\{111\}$ facets. Moreover, it is conceivable that the energy difference between $\{100\}$ and $\{111\}$ facets will increase with the increase of concentration of $\mathrm{Pt}$ atoms in nanoparticles. Hence, compared to the octahedral nanoparticles solely terminated with $\{111\}$ facets, the cubo-octahedral nanoparticles 
terminated with $\{111\}$ and $\{100\}$ facets could have lower energy for $\mathrm{Pt}_{50} \mathrm{Ni}_{50}$ but higher energies for $\mathrm{Pt}_{75} \mathrm{Ni}_{25}$. In addition, the energy difference between cubic and tetrahedral $\mathrm{Pt}_{75} \mathrm{Ni}_{25}$ nanoparticles is larger than for $\mathrm{Pt}_{50} \mathrm{Ni}_{50}$ nanoparticles.

\section{B. Segregation in relaxed Pt-Ni nanoparticles}

For bimetallic alloys (like Pt-Ni) in which the two component elements have a large difference in atom size, atomic relaxation is an important factor in determining surface segregation and equilibrium shape of its nanoparticles. Next, we will consider the effect of relaxation on segregation in Pt-Ni nanoparticles. Since the cubic and tetrahedral nanoparticles are found to have much higher energy (see Fig. 2) and thus are meta-stable configurations, we only carried out relaxation studies for the octahedral and cubooctahedral Pt-Ni nanoparticles in this work. The Pt-Ni nanoparticles initially had the lattice constants determined for the bulk alloys at the same composition and temperature $600 \mathrm{~K}$ (see details in Sec. II.C) with randomly distributed Pt and Ni atoms. For each nanoparticle, we performed $\mathrm{MC}$ simulations allowing both atomic displacement and exchange of element types for 40 million $\mathrm{MC}$ steps at $\mathrm{T}=600 \mathrm{~K}$. During the $\mathrm{MC}$ simulations, both the lattice constants and the distribution of $\mathrm{Pt}$ and $\mathrm{Ni}$ atoms in the nanoparticles change. Due to larger inward relaxations at the vertices and edges compared to the rest of the surface, the relaxed nanoparticles are more round than the unrelaxed ones. As a result, the different surface sites (facet, edge, and vertex) are less distinguishable in the relaxed nanoparticles. Moreover, we found the following two distinct features for Pt-Ni nanoparticles.

\section{1. $\{100\}$-facet reconstruction in the cubo-octahedral Pt-Ni nanoparticles}

We found that a reconstruction process often occurs in $\{100\}$ facets of relaxed cubo-octahedral Pt-Ni nanoparticles during the MC simulations. In Fig. 3, we show the final configurations of the $\mathrm{Pt}_{50} \mathrm{Ni}_{50}$ (Fig. 3(a)) and $\mathrm{Pt}_{75} \mathrm{Ni}_{25}$ (Fig. 3(b)) cubo-octahedral nanoparticles after 40 million MC steps. Compared to the original cubo-octahedral nanoparticle (shown in Fig. 1(d)), the $4 \times 4$ atoms in the (100) facet outlined by dashed lines have moved significantly away from their original positions. In fact, they tend to arrange themselves into a denser hexagonal configuration together with some atoms coming from the core of the nanoparticles. The external edges of the $4 \times 4$ array are intact 
but distorted away from a square shape, especially obvious in Fig. 3(b) for the $\mathrm{Pt}_{75} \mathrm{Ni}_{25}$ nanoparticle. The reconstruction of $\{100\}$ facets from a square to a hexagonal lattice in the surface of nanoparticles is reasonable, since it has been observed for Pt-Ni alloys that the top layer of the (100) surface can reconfigure to such a hexagonal arrangement of atoms $[17,18]$.

One may wonder whether the energy lowering associated with the $\{100\}$-facet reconstruction, as well as the other relaxations, might be sufficient to favor the cubooctahedral shape over the octahedral shape. After all, high-resolution electron microscopy (HREM) suggests a cubo-octahedral shape [11,49]. Our calculations also find this to be the case, but only by a very small energy difference on the order of $0.001 \mathrm{eV} /$ atom. However, even if there were a larger energy difference, we must be careful before drawing conclusions about which nanoparticle shape or shapes should be observed experimentally.

First, most HREM experiments are performed at ambient pressures that almost guarantee a degree of surface contamination on the nanoparticle surface, for example by $\mathrm{CO}$ molecules and carbon atoms. Furthermore, the substrate materials for the imaging of such nanoparticles, such as carbon [11] and $\mathrm{SiO}_{2}$ [49], most likely also contribute contamination to the particle surfaces. Any such contamination probably removes the surface reconstruction, as is well known from many experiments on extended surfaces. Therefore, any energy gain due to $\{100\}$-facet reconstruction will probably only matter under more idealized vacuum conditions.

Second, our simulations are performed for nanoparticles with magic numbers of atoms. These magic numbers change from the octahedral to the cubo-octahedral shape, so that for a given number of atoms, one shape may be magic (with complete atomic shells) while the other is not (having incomplete shells), making the comparison risky. Furthermore, any given real nanoparticle most likely does not have a magic number of atoms (for any shape), so that its outermost atomic shell would be incomplete. Incomplete shells imply defects, such as missing corner or edge atoms, islands on facets, steps surrounding islands, etc. These defects cost energy and should be considered when comparing shapes. 
Third, the $\{100\}$-facet reconstruction that we predict is created at the expense of vacancy defects inside the nanoparticles. Besides making the magic-number property unclear (external as well as internal shells are now incomplete), this would require lengthy further "annealing" to explore whether those internal defects can be eliminated or are an inevitable consequence of the surface reconstruction.

Fourth, the shape of a nanoparticle, at any temperature, must vary dynamically as atoms move around, since the energy differences between configurations are very small compared to $k_{\mathrm{B}} \mathrm{T}$. There may be an optimum lowest-energy shape in the absence of vibrations, but thermal energy will favor a much wider family of shapes.

These aspects are beyond the scope of the present work.

\section{Surface-sandwich structure of Pt-Ni nanoparticles}

In Fig. 4, we show cross-sections that expose the centers of the Pt-Ni nanoparticles containing about 600 atoms. These plots qualitatively reveal a "surfacesandwich structure" for the equilibrium Pt-Ni nanoparticles regardless of their shape and composition. The Pt atoms are enriched in the outermost and third atomic shells, while the Ni atoms are enriched in the second atomic shell. This surface-sandwich structure in nanoparticles terminated with $\{111\}$ and $\{100\}$ facets is consistent with the oscillatory segregation behavior known for the extended (111) and (100) surfaces of Pt-Ni alloys. Table VIII reports our calculated concentrations of Pt atoms $\left(\mathrm{C}_{\mathrm{Pt}_{\mathrm{t}}}\right)$ in the outermost three atomic shells and inner core of $\mathrm{Pt}_{50} \mathrm{Ni}_{50}$ nanoparticles. Table IX gives the corresponding results for $\mathrm{Pt}_{75} \mathrm{Ni}_{25}$ nanoparticles. To eliminate the influence of the original structure, we calculated the concentrations of $\mathrm{Pt}$ atoms by averaging values sampled every $10000 \mathrm{MC}$ steps in the last 20 million MC steps of simulations.

For octahedral $\mathrm{Pt}_{50} \mathrm{Ni}_{50}$ nanoparticles, the surface-sandwich structures are shown clearly in Table VIII. The Pt concentration is above 72 at.\% in the outermost atomic shell, below 24 at.\% in the second atomic layer, and about 42 at.\% (higher than 36 at.\% found in the inner core) in the third atomic shell.. The segregation profiles of nanoparticles are close to the segregation profiles in Table VI for extended (111) surfaces. The observed slight increase of the Pt concentration with nanoparticle size is a geometrical effect due to the decreasing surface-to-volume ratio when the nanoparticle 
size increases: the number of Pt atoms grows faster with particle size than the surface area to which they can segregate to.

The surface-sandwich structure is also formed for cubo-octahedral $\mathrm{Pt}_{50} \mathrm{Ni}_{50}$ nanoparticles containing fewer than 2000 atoms. However, the equilibrium structures of cubo-octahedral $\mathrm{Pt}_{50} \mathrm{Ni}_{50}$ nanoparticles containing more than 2000 atoms are more like a core-shell structure: Pt is enriched in the outermost shell and depleted in the homogenous core. We believe that the strong ordering tendency in the core region of larger nanoparticles accounts for this behavior. For bulk $\mathrm{Pt}_{50} \mathrm{Ni}_{50}$ alloys, the disordered fcc structure will change to the ordered $\mathrm{L} 1_{0}$ structure below $900 \mathrm{~K}$ [50]. It is known that the order-disorder transition temperature of nanoparticles is a function of their size and shape $[51,52]$. The transition temperature is higher for larger nanoparticles, therefore, the simulation temperature of $600 \mathrm{~K}$ might be below the order-disorder transition temperatures of larger cubo-octahedral $\mathrm{Pt}_{50} \mathrm{Ni}_{50}$ nanoparticles while above the orderdisorder transition temperatures of smaller cubo-octahedral $\mathrm{Pt}_{50} \mathrm{Ni}_{50}$ nanoparticles. We indeed observed ordered PtNi clusters in the core of the two simulated larger nanoparticles.

It was found experimentally that the catalytic behavior of the samples, which contain cubo-octahedral $\mathrm{Pt}_{50} \mathrm{Ni}_{50}$ nanoparticles with a diameter from 2 to $6 \mathrm{~nm}$, varies dramatically for different samples in oxygen reduction reaction (ORR) [11]. We propose that this may be due to a mixture of smaller nanoparticles that assume a surface-sandwich structure and larger nanoparticles that assume a core-shell structure with an ordered core: these different structures might cause them to behave differently in the ORR environment.

Table IX indicates that both octahedral and cubo-octahedral $\mathrm{Pt}_{75} \mathrm{Ni}_{25}$ nanoparticles adopt the surface-sandwich structures. In these nanoparticles, the Pt concentration in the outermost atomic shell is about 50 at.\% higher than in the second atomic shell; and the Pt concentration in the third atomic shell is about 75 at.\%. It is found that the $\mathrm{Pt}$ concentration is nearly 100 at.\% in the outermost layer of $\mathrm{Pt}_{75} \mathrm{Ni}_{25}$ nanoparticles. The formation of a Pt "skin" covering the core of nanoparticles is of great significance, because it guarantees a maximum exposure of active catalyst Pt to reactants. The 
concentrations of $\mathrm{Pt}$ atoms in the sub-layer (second atomic shell) of $\mathrm{Pt}_{75} \mathrm{Ni}_{25}$ nanoparticles are about 25 at.\% smaller than the overall concentration of 75 at.\% and increase gradually with their size. This implies that the $\mathrm{Ni}$ atoms are enriched in the sub-layer of nanoparticles. Even better, the extent of enrichment is controllable by varying the nanoparticle size. It is widely believed that modification of the electronic structure of $\mathrm{Pt}$ by neighboring transition metal $\mathrm{Ni}$ atoms is a major reason why alloying enhances catalytic performance for Pt-bimetallic surfaces [53]. Therefore, our study points out that controlling the nanoparticle size can tailor the electronic structure of $\mathrm{Pt}$ in the nanoparticle surfaces, besides varying the type of alloying element and the overall composition.

\section{CONCLUSIONS}

We have investigated segregation phenomena in disordered fcc $\mathrm{Pt}_{50} \mathrm{Ni}_{50}$ and $\mathrm{Pt}_{75} \mathrm{Ni}_{25}$ catalyst nanoparticles, using MEAM potentials and Monte Carlo method. The developed MEAM potentials successfully reproduced the main features, which are oscillatory segregation profiles and segregation reversal in (110) surfaces, of the observed segregation phenomena in extended low-index surfaces for disordered Pt-Ni alloys. Moreover, our simulations predict that a surface reconstruction from a square lattice to a denser hexagonal lattice occurs in the top layer of Pt-Ni alloy (100) surfaces, agreeing with experimental measurements [17,18]. Therefore, our employed MEAM potentials and Monte Carlo method are suitable for studying segregation in Pt-Ni nanoparticles.

In this work, we assume that $\mathrm{Pt}-\mathrm{Ni}$ nanoparticles have disordered fcc configurations based on previous HREM results [11]. Among four possible kinds (cube, tetrahedron, octahedron, and cubo-octahedron) of nanoparticles, we find for Pt-Ni alloys that the octahedral nanoparticles (terminated with $\{111\}$ facets) and cubo-octahedral nanoparticles (terminated with $\{111\}$ and $\{100\}$ facets) have lowest and nearly equal energies. In contrast, the Pt-Ni nanoparticles are most frequently found to be cubooctahedral $[11,49]$. For this discrepancy between theory and experiments, we argue that due to reconstruction processes in $\{100\}$ facets the simulated cubo-octahedral nanoparticles are of some defects and thus higher energies. A "perfect" $\{100\}$-facets reconstructed cubo-octahedral nanoparticle should be most energetically favorable. 
More importantly, we predict a surface-sandwich structure for most equilibrium Pt-Ni nanoparticles regardless of their shape and composition. In the surface sandwich structure of Pt-Ni nanoparticles, the Pt atoms are enriched in the outermost and third atomic shells while the $\mathrm{Ni}$ atoms are enriched in the second atomic shell. For $\mathrm{Pt}_{50} \mathrm{Ni}_{50}$ cubo-octahedral nanoparticles containing more than 2000 atoms, we find a core-shell structure, in which the concentrations of Pt atoms in the second atomic shell and beneath inside nanoparticles are very close, instead of the surface-sandwich structure. We believe the stronger ordering tendency in the larger $\mathrm{Pt}_{50} \mathrm{Ni}_{50}$ cubo-octahedral nanoparticles accounts for this morphological transition. In either structure, the Pt atoms are significantly enriched in the outermost atomic shell of Pt-Ni nanoparticles. These results indicate an economical design of $\mathrm{Pt}-\mathrm{Ni}$ catalysts: arrange the precious catalyst $\mathrm{Pt}$ predominately at the outer surfaces of nanoparticles by surface segregation process. Therefore, this work should be useful for the future processing, improvement, and design of Pt-Ni catalyst nanoparticles.

\section{ACKNOWLEDGMENTS}

We are grateful to Dr. A. Canning and Dr. J. An for assistance in using the PARATEC code. This work was supported by the Office of Science, Materials Sciences Division, of the U.S. Department of Energy under Contract Nos. DE-AC03-76SF00098 at LBNL and W-7405-ENG-36 at LANL. The computations were carried out at the National Energy Research Scientific Computing Center (NERSC), which is operated by LBNL for the U.S. Department of Energy. 


\section{References:}

[1] G.A. Somorjai and Y.G. Borodko, Catal. Lett. 1, 76 (2001).

[2] J.H. Sinfelt, Acc. Chem. Res. 10, 15 (1977).

[3] J.H. Sinfelt, Rev. Mod. Phys. 51, 569 (1979).

[4] C.T. Campbell, Annu. Rev. Phys. Chem. 41, 775 (1990).

[5] J.K Strohl and T.S. King, J. Catal. 116, 540 (1989).

[6] D.S. Mainardi and P.B. Baluena, Langmuir 17, 2047 (2001).

[7] E. Christoffersen, D. Stoltze, and J.K. Nørskov, Surf. Sci. 505, 200 (2002).

[8] S. Helfensteyn and C. Creemers, Surf. Sci. 507-510, 783 (2002).

[9] C. Mottet, G. Tréglia, and B. Legrand, Phys. Rev. B 66, 045413 (2002).

[10] V. Stamenković, T.J. Schmidt, P.N. Ross, and N.M. Marković, J. Phys. Chem. B 106, 11970 (2002).

[11] U.A. Paulus, A. Wokaun, G.G. Scherer, T.J. Schmidt, V. Stamenković, V. Radmilovic, N.M. Marković, and P.N. Ross, J. Phys. Chem. B 106, 4181 (2002).

[12] Y. Gauthier, Y. Joly, R. Baudoing, and J. Rundgren, Phys. Rev. B 31, 6216 (1985).

[13] Y. Gauthier, R. Baudoing, M. Lundberg and J. Rundgren, Phys. Rev. B 35, 7867 (1987).

[14] Y. Gauthier, R. Baudoing, and J. Jupille, Phys. Rev. B 40, 1500 (1989).

[15] Y. Gauthier and R. Baudoing, Surface Segregation Phenomena, Eds. P.A. Dowben and A. Miller (CRC press, Boca Raton, FL, 1990), p.169.

[16] S. Deckers, F.H.P.M. Habraken, W.F. van der Weg, A.W. Denier van der Gon, B. Pluis, J.F. van der Veen, and R. Baudoing, Phys. Rev. B 42, 3253 (1990).

[17] Y. Gauthier, R. Baudoing-Savois, J. Rundgren, M. Hammar, and M. Gothelid, Surf. Sci. 327, 100 (1995).

[18] W. Hebenstreit, G. Ritz, M. Schmid, A. Biedermann, and P. Varga, Surf. Sci. 388, 150 (1997).

[19] M. Lundberg, Phys. Rev. B 36, 4692 (1987).

[20] B. Legrand, G. Tréglia and F. Ducastelle, Phys. Rev. B 41, 4422 (1990).

[21] H. Stadler, W. Hofer, M. Schmid, and P. Varga, Surf. Sci. 287, 366 (1993).

[22] I.A. Abrikosov, A.V. Ruban, H.L. Skriver and B. Johansson, Phys. Rev. B 50, 2039 (1994). 
[23] P. Deurinck and C. Creemers, Surf. Sci. 441, 493 (1999).

[24] L.V. Pourovskii, A.V. Ruban, I.A. Abrikosov, Y.Kh. Vekilov, and B. Johansson, Phys. Rev. B 64, 035421 (2001).

[25] M.I. Baskes, Phys. Rev. B 62, 2727 (1992).

[26] S.M. Foiles, M.I. Baskes, and M.S. Daw, Phys. Rev. B 33, 7983 (1986).

[27] M.I. Baskes, Mater. Sci. Eng., A 261, 165 (1999).

[28] M.I. Baskes, Mater. Chem. Phys. 50, 152 (1997).

[29] J.H. Rose, J.R. Smith, F. Guinea, and J. Ferrante, Phys. Rev. B 29, 2963 (1984).

[30] N. Metropolis, A.W. Rosenbluth, M.N. Rosenbluth, A.H. Teller, and E. Teller, J. Chem. Phys. 21, 1087 (1953).

[31] S.M. Foiles, Surface Segregation Phenomena, Eds. P.A. Dowben and A. Miller (CRC press, Boca Raton, FL, 1990), p.79.

[32] W.B. Pearson, A Handbook of Lattice Spacings and Structures of Metals and Alloys, (Pergamon, Oxford, 1964), p. 782.

[33] M.I. Baskes, J.E. Angelo, and C.L. Bisson, Modell. Simul. Mater. Sci. Eng. 2, 505 (1994).

[34] L. Vitos, A.V. Ruban, H.L. Skriver, and J. Kollár, Surf. Sci. 411, 186 (1998).

[35] W.R. Tyson and W.A. Miller, Surf. Sci. 62, 267 (1977).

[36] F.R. de Boer, R.Room, W.C. Mattens, A.R. Miedema, A.K. Niessen, Cohesion in Metals (North-Holland, Amsterdam, 1988).

[37] B.G. Pfrommer, J. Demmel, and H. Simon, J. Comp. Phys. 150, 287 (1999); B. G. Pfrommer, M. Coté, S. G. Louie, and M. L. Cohen, J. Comp. Phys. 131, 233 (1997); also see details at website http://www.nersc.gov/projects/paratec.

[38] M. Fuchs and M. Scheffler, Comput. Phys. Commun. 119, 67 (1999).

[39] N. Troullier and J. L. Martins, Phys. Rev. B 43, 1993 (1991).

[40] P. Söderlind, O. Eriksson, J.M. Wills, and A.M. Boring, Phys. Rev. B 48, 5844 (1993).

[41] A.V. Ruban, H.L. Skriver, and J.K. Nørskov, Phys. Rev. B 59, 15990 (1999).

[42] R. Baudoing, Y. Gauthier, M. Lundberg, and J. Rundgren, J. Phys. C 19, 2825 (1986). 
[43] S. Deckers, F.H.P.M. Habraken, W.F. van der Weg, A.W. Denier van der Gon, B. Pluis, J.F. van der Veen, and R. Baudoing, Phys. Rev. B 42, 3253 (1990).

[44] A. Pantförder, J. Skonieczny, E. Janssen, G. Meister, A. Goldmann, and P. Varga, Surf. Sci. 337, 177 (1995).

[45] T.S. Ahmadi, Z.L. Wang, T.C. Green, A. Henglein, and M.A. El-Sayed, Science 272, 1924 (1996).

[46] R. Van Hardeveld and F. Hartog, Surf. Sci. 15, 189 (1969).

[47] C.L. Cleveland and U. Landman, J. Chem. Phys. 94, 7376 (1991).

[48] J. Uppenbrink and D.J. Wales, J. Chem. Phys. 96, 8520 (1992).

[49] J. Arenas-Alatorre, M. Avalos-Borja, and G. Díaz, Appl. Surf. Sci. 189, 7 (2002).

[50] C.E. Dahmani, M.C. Cadeville, J.M. Sanchez, and J.L Morán-Lópes, Phys. Rev. Lett. 55, 1208( 1985).

[51] T. Tadaki, A. Koreeda, Y. Nakata, and T. Kinoshita, Surf. Rev. Lett. 3, 65 (1996).

[52] T. Tadaki, T. Kinoshita, Y. Nakata, T. Ohkubo, and Y. Hirotsu, Z. Phys. D 40, 493 (1997).

[53] T. Jacob, B.V. Merinov, and W.A. Goddard, Chem. Phys. Lett. 385, 374 (2004). 
TABLE I. Parameters for the MEAM potentials of Pt, Ni and Pt-Ni. The parameters are: the cohesive energy $E_{c}(e V)$, the equilibrium nearest-neighbor distance $r_{e}(\AA)$, the exponential decay factor for the universal energy function $\alpha$, the scaling factor for the embedding energy A, the four exponential decay factors for the atomic densities $\beta^{(i)}$, the four weighting factors for the atomic densities $\mathrm{t}^{(\mathrm{i})}$, and the density scaling factor $\rho^{0}$.

\begin{tabular}{lccccccccccccc}
\hline \hline & $\mathrm{E}_{\mathrm{c}}$ & $\mathrm{r}_{\mathrm{e}}$ & $\alpha$ & $\mathrm{A}$ & $\beta^{(0)}$ & $\beta^{(1)}$ & $\beta^{(2)}$ & $\beta^{(3)}$ & $\mathrm{t}^{(0)}$ & $\mathrm{t}^{(1)}$ & $\mathrm{t}^{(2)}$ & $\mathrm{t}^{(3)}$ & $\rho^{0}$ \\
\hline $\mathrm{Ni}$ & 4.45 & 2.49 & 4.99 & 1.10 & 2.45 & 1.50 & 6.00 & 1.50 & 1.00 & 5.79 & 1.60 & 3.70 & 1.00 \\
$\mathrm{Pt}$ & 5.77 & 2.77 & 6.44 & 1.04 & 4.673 & 2.20 & 6.00 & 2.20 & 1.00 & 4.70 & -1.38 & 3.29 & 1.10 \\
$\mathrm{PtNi}$ & 5.52 & 2.70 & 6.43 & - & - & - & - & - & - & - & - & - & - \\
\hline \hline
\end{tabular}

TABLE II. Angular screening parameters for the MEAM potentials.

\begin{tabular}{lcccccc}
\hline \hline & Pt-Pt-Pt & Pt-Ni-Pt & Ni-Pt-Pt & Ni-Ni-Pt & Ni-Pt-Ni & Ni-Ni-Ni \\
\hline $\mathrm{C}_{\max }$ & 2.8 & 2.8 & 2.8 & 2.8 & 2.8 & 2.8 \\
$\mathrm{C}_{\min }$ & 0.8 & 2.0 & 1.0 & 1.0 & 0.8 & 0.8 \\
\hline \hline
\end{tabular}

TABLE III. Energies of the relaxed extended low-index fcc surfaces for pure Pt and pure Ni calculated using the MEAM potentials, compared to the GGA-DFT and experimental results.

\begin{tabular}{llccc}
\hline \hline element & surfaces & MEAM $\left(\mathrm{mJ} / \mathrm{m}^{2}\right)$ & GGA-DFT $^{\mathrm{a}}\left(\mathrm{mJ} / \mathrm{m}^{2}\right)$ & Experiment $\left(\mathrm{mJ} / \mathrm{m}^{2}\right)$ \\
\hline $\mathrm{Pt}$ & $(111)$ & 1651 & 2299 & $2489^{\mathrm{b}}, 2475^{\mathrm{c}}$ \\
& $(100)$ & 2155 & 2734 & \\
& $(110)$ & 1983 & 2819 & $2380^{\mathrm{b}}, 2450^{\mathrm{c}}$ \\
$\mathrm{N} \mathrm{Ni}$ & $(111)$ & 2039 & 2011 & \\
& $(100)$ & 2438 & 2426 & \\
& $(110)$ & 2362 & 2368 & \\
\end{tabular}

${ }^{\mathrm{a}}$ Reference [34].

${ }^{\mathrm{b}}$ Reference [35].

${ }^{\mathrm{c}}$ Reference [36]. 
TABLE IV. Comparison of the calculated properties of bulk $\mathrm{Pt}_{3} \mathrm{Ni}\left(\mathrm{Ll}_{2}\right)$, $\mathrm{PtNi}\left(\mathrm{L}_{0}\right)$, and $\mathrm{PtNi}_{3}\left(\mathrm{Ll}_{2}\right)$ using the MEAM potentials and the ab initio (LDA-DFT) method.

\begin{tabular}{|c|c|c|c|c|c|c|c|c|c|}
\hline & & & & \multicolumn{3}{|c|}{ MEAM } & \multicolumn{3}{|c|}{ LDA-DFT } \\
\hline \multicolumn{10}{|c|}{ Properties of $\mathrm{Pt}_{3} \mathrm{Ni}\left(\mathrm{Ll}_{2}\right)$} \\
\hline \multicolumn{4}{|c|}{ Lattice constant a $(\AA)$} & \multicolumn{3}{|c|}{3.819} & \multicolumn{3}{|c|}{3.820} \\
\hline \multicolumn{4}{|c|}{ Cohesive energy (eV/atom) } & \multicolumn{3}{|c|}{-0.080} & \multicolumn{3}{|c|}{-0.079} \\
\hline \multicolumn{4}{|c|}{ Elastic constant B $(\mathrm{GPa})$} & \multicolumn{3}{|c|}{291.7} & \multicolumn{3}{|c|}{291.7} \\
\hline \multicolumn{4}{|c|}{ Elastic constant $\left(\mathrm{C}_{11}-\mathrm{C}_{22}\right) / 2(\mathrm{GPa})$} & \multicolumn{3}{|c|}{62.3} & \multicolumn{3}{|c|}{66.0} \\
\hline \multicolumn{4}{|c|}{ Elastic constant $\mathrm{C}_{44}(\mathrm{GPa})$} & \multicolumn{3}{|c|}{102.7} & \multicolumn{3}{|c|}{123.4} \\
\hline \multicolumn{10}{|c|}{ Properties of PtNi $\left(\mathrm{Ll}_{0}\right)^{\mathrm{a}}$} \\
\hline \multicolumn{4}{|c|}{ Lattice constant a $(\AA)$} & \multicolumn{3}{|c|}{3.790} & \multicolumn{3}{|c|}{3.794} \\
\hline \multicolumn{4}{|c|}{ Lattice constant c $(\AA)$} & \multicolumn{3}{|c|}{3.601} & \multicolumn{3}{|c|}{3.574} \\
\hline \multicolumn{4}{|c|}{ Cohesive energy (eV/atom) } & \multicolumn{3}{|c|}{-0.156} & \multicolumn{3}{|c|}{-0.117} \\
\hline \multicolumn{10}{|c|}{ Properties of $\mathrm{PtNi}_{3}\left(\mathrm{Ll}_{2}\right)$} \\
\hline \multicolumn{4}{|c|}{ Lattice constant a $(\AA)$} & \multicolumn{3}{|c|}{3.632} & \multicolumn{3}{|c|}{3.607} \\
\hline \multicolumn{4}{|c|}{ Cohesive energy (eV/atom) } & \multicolumn{3}{|c|}{-0.144} & & -0.089 & \\
\hline $\begin{array}{l}\mathrm{a} \text { The exp } \\
\mathrm{c}=3.582\end{array}$ & $\begin{array}{l}\text { mental } \\
\mathrm{T}=88\end{array}$ & $\begin{array}{l}\text { attice } 1 \\
\mathrm{~K} \text {. }\end{array}$ & cameter & for $\mathrm{Ptl}$ & $\left(\mathrm{L}_{0}\right)$ & om Ref & [32] ar & $\mathrm{a}=3.81$ & $\AA ̊$ and \\
\hline $\begin{array}{l}\text { TABLE } \\
\text { first, seco }\end{array}$ & $\begin{array}{l}\text { Segres } \\
\text { and thi }\end{array}$ & $\begin{array}{l}\text { ition er } \\
\text { d layer }\end{array}$ & $\begin{array}{l}\text { gies (i } \\
\text { the ho }\end{array}$ & $\begin{array}{l}\mathrm{eV}) \text { of } \\
(\mathrm{Pt} \text { or }\end{array}$ & $\begin{array}{l}\text { he singl } \\
\text { i) surfac }\end{array}$ & atom i & purity & $\mathrm{Ni}$ or $\mathrm{P}$ & on the \\
\hline & & $(111)$ & & & $(100)$ & & & $(110)$ & \\
\hline & 1 & 2 & 3 & 1 & 2 & 3 & 1 & 2 & 3 \\
\hline $\mathrm{Ni}$ on $\mathrm{Pt}$ & 0.27 & -0.12 & -0.01 & -0.07 & -0.18 & 0.00 & -0.19 & -0.02 & -0.03 \\
\hline $\mathrm{Pt}$ on $\mathrm{Ni}$ & -0.35 & 0.01 & -0.01 & -0.22 & -0.02 & -0.01 & -0.09 & -0.23 & -0.02 \\
\hline
\end{tabular}


TABLE VI. Segregation concentration profile for extended surfaces of disordered $\mathrm{Pt}_{50} \mathrm{Ni}_{50}$ alloys simulated using our MEAM potentials at $\mathrm{T}=1200 \mathrm{~K}$. Three different choices for the interlayer spacing are used: a non-relaxed surface, a surface with relaxation determined by $\mathrm{LEED}$, and a relaxation from the $\mathrm{MC}$ simulations allowing atom displacements normal to the surface. The calculated segregation profiles for $\mathrm{Pt}_{50} \mathrm{Ni}_{50}$ alloys are compared with first-principles and experimental results. $\mathrm{C}_{\mathrm{i}}(\mathrm{i}=1,2,3)$ is the $\mathrm{Pt}$ concentration in layer $i$ and in atomic percent. $\Delta_{12}$ and $\Delta_{23}$ give the spacing relaxations (in percent), relative to the perfect crystal, between the first and second layers and between the second and third layers, respectively. Positive (negative) values of $\Delta_{12}$ and $\Delta_{23}$ signify expansions (contractions).

\begin{tabular}{|c|c|c|c|c|c|c|}
\hline & & $\mathrm{C}_{1}$ & $\mathrm{C}_{2}$ & $\mathrm{C}_{3}$ & $\Delta_{12}$ & $\Delta_{23}$ \\
\hline \multirow[t]{5}{*}{$(111)$} & No relaxation & 77 & 26 & 59 & 0.0 & 0.0 \\
\hline & Relaxation from LEED & 79 & 19 & 61 & -2.0 & -2.0 \\
\hline & Relaxation from simulation & 80 & 31 & 53 & 1.9 & -1.6 \\
\hline & First-principles calculation ${ }^{a}$ & 70 & 37 & 57 & - & - \\
\hline & LEED study $^{\text {b }}$ & $88( \pm 2)$ & $9( \pm 5)$ & $65( \pm 2)$ & -2.0 & -2.0 \\
\hline \multirow[t]{5}{*}{$(100)$} & No relaxation & 70 & 28 & 65 & 0.0 & 0.0 \\
\hline & Relaxation from LEED & 79 & 22 & 52 & 4.6 & -9.0 \\
\hline & Relaxation from simulation & 66 & 29 & 62 & -2.9 & -2.0 \\
\hline & First-principles calculation ${ }^{a}$ & 87 & 23 & 70 & - & - \\
\hline & LEED study $^{b}$ & $86( \pm 10)$ & $24( \pm 10)$ & & 4.6 & -9.0 \\
\hline \multirow[t]{5}{*}{$(110)$} & No relaxation & 49 & 66 & 39 & 0.0 & 0.0 \\
\hline & Relaxation from LEED & 7 & 93 & 40 & -19.2 & 10.5 \\
\hline & Relaxation from simulation & 20 & 90 & 22 & -19.8 & 8.1 \\
\hline & First-principles calculation ${ }^{\text {a }}$ & 7 & 100 & 20 & - & - \\
\hline & LEED study $^{\text {b }}$ & $0( \pm 6)$ & $95( \pm 4)$ & $17( \pm 7)$ & -19.2 & 10.5 \\
\hline
\end{tabular}

\footnotetext{
${ }^{\mathrm{a}}$ Reference [22].

${ }^{\mathrm{b}}$ Reference [15].
} 
TABLE VII. Segregation concentration profile for extended surfaces of disordered $\mathrm{Pt}_{75} \mathrm{Ni}_{25}$ alloys simulated using our MEAM potentials at $\mathrm{T}=1200 \mathrm{~K}$. $\mathrm{C}_{\mathrm{i}}(\mathrm{i}=1,2,3)$ is the $\mathrm{Pt}$ concentration in layer $i$ and in atomic percent. $\Delta_{12}$ and $\Delta_{23}$ give the spacing relaxations (in percent), relative to the perfect crystal, between the first and second layers and between the second and third layers, respectively. Positive (negative) values of $\Delta_{12}$ and $\Delta_{23}$ signify expansions (contractions).

\begin{tabular}{lllllll}
\hline \hline & & $\mathrm{C}_{1}$ & $\mathrm{C}_{2}$ & $\mathrm{C}_{3}$ & $\Delta_{12}$ & $\Delta_{23}$ \\
\hline \multirow{2}{*}{$(111)$} & No relaxation & 97 & 51 & 85 & 0.0 & 0.0 \\
& Relaxation from simulation & 97 & 53 & 81 & 1.1 & -1.9 \\
\hline \multirow{2}{*}{$(100)$} & No relaxation & 89 & 59 & 83 & 0.0 & 0.0 \\
& Relaxation from simulation & 82 & 55 & 81 & -3.9 & -2.5 \\
\hline \multirow{2}{*}{$(110)$} & No relaxation & 79 & 83 & 69 & 0.0 & 0.0 \\
& Relaxation from simulation & 59 & 92 & 56 & -23.4 & 11.8 \\
\hline \hline
\end{tabular}

Table VIII. Atomic concentrations of Pt atoms in the outermost layer (denoted $\mathrm{C}_{1}$ ), the second layer (denoted $\mathrm{C}_{2}$ ), the third layer (denoted $\mathrm{C}_{3}$ ), and the remainder (denoted $\mathrm{C}_{\text {core }}$ ) of $\mathrm{Pt}_{50} \mathrm{Ni}_{50}$ nanoparticles at $\mathrm{T}=600 \mathrm{~K}$.

\begin{tabular}{lcccc}
\hline \hline Number of atoms & $\mathrm{C}_{1}$ & $\mathrm{C}_{2}$ & $\mathrm{C}_{3}$ & $\mathrm{C}_{\text {core }}$ \\
\hline Octahedral nanoparticles & & & & \\
670 & 72 & 20 & 43 & 37 \\
1156 & 75 & 23 & 43 & 36 \\
2255 & 80 & 29 & 39 & 36 \\
3894 & 83 & 29 & 42 & 38 \\
\hline Cubo-octahedral nanoparticles & & & & \\
586 & 70 & 27 & 44 & 35 \\
1289 & 74 & 31 & 43 & 35 \\
2406 & 79 & 36 & 38 & 37 \\
4033 & 81 & 37 & 41 & 39 \\
\hline \hline
\end{tabular}


Table IX. Atomic concentrations of Pt atoms in the outermost layer (denoted $\mathrm{C}_{1}$ ), the second layer (denoted $\mathrm{C}_{2}$ ), the third layer (denoted $\mathrm{C}_{3}$ ), and the remainder (denoted $\mathrm{C}_{\text {core }}$ ) of $\mathrm{Pt}_{75} \mathrm{Ni}_{25}$ nanoparticles at $\mathrm{T}=600 \mathrm{~K}$.

\begin{tabular}{lllll}
\hline \hline Number of atoms & $\mathrm{C}_{1}$ & $\mathrm{C}_{2}$ & $\mathrm{C}_{3}$ & $\mathrm{C}_{\text {core }}$ \\
\hline Octahedral nanoparticles & & & & \\
670 & 98 & 39 & 77 & 61 \\
1156 & 98 & 43 & 78 & 65 \\
2255 & 99 & 50 & 74 & 67 \\
3894 & 99 & 55 & 70 & 69 \\
\hline Cubo-octahedral nanoparticles & & & & \\
586 & 97 & 44 & 75 & 59 \\
1289 & 99 & 48 & 78 & 62 \\
2406 & 99 & 55 & 75 & 64 \\
4033 & 99 & 58 & 74 & 67 \\
\hline \hline
\end{tabular}




\section{Figure Captions:}

FIG. 1. (a) - (d): Structures of cubic, tetrahedral, octahedral, and cubo-octahedral nanoparticles, respectively; (e): Numbering of nearest neighbors in fcc structure.

FIG. 2. Plot of the approximately linear relation between the atomic cohesive energy $\mathrm{U} / \mathrm{N}$ (in $\mathrm{eV}$ ) of cubic (circles), tetrahedral (squares), octahedral (up-triangles), and cubo-octahedral (down-triangles) nanoparticles and the scaled number of atoms $\mathrm{N}^{-1 / 3}$ of nanoparticles. (a) $\mathrm{Pt}_{50} \mathrm{Ni}_{50}$ alloys. (b) $\mathrm{Pt}_{75} \mathrm{Ni}_{25}$ alloys.

FIG. 3. Snapshots of the equilibrium fcc cubo-octahedral nanoparticle (containing 586 atoms) simulated at $\mathrm{T}=600 \mathrm{~K}$. (a) $\mathrm{Pt}_{50} \mathrm{Ni}_{50}$ alloys. (b) $\mathrm{Pt}_{75} \mathrm{Ni}_{25}$ alloys. In these panels, the open circles represent the Pt atoms and the gray circles stand for the $\mathrm{Ni}$ atoms. As a guide to the eye, the edges of one reconstructed $\{100\}$ facet are delineated with dashed lines. Note that the atoms in the reconstructed facet are more coplanar than appears in this rendition, as can be seen in the similar facets at top and right.

FIG. 4. A few [001] cross-sectional views of the surface-sandwich structure of Pt-Ni nanoparticles simulated at $\mathrm{T}=600 \mathrm{~K}$. These panels show (a) octahedral $\mathrm{Pt}_{50} \mathrm{Ni}_{50}$ nanoparticle (containing 670 atoms), (b) cubo-octahedral $\mathrm{Pt}_{50} \mathrm{Ni}_{50}$ nanoparticle (containing 586 atoms), (c) octahedral $\mathrm{Pt}_{75} \mathrm{Ni}_{25}$ nanoparticle (containing 670 atoms), and (d) cubo-octahedral $\mathrm{Pt}_{75} \mathrm{Ni}_{25}$ nanoparticle (containing 586 atoms). The open circles represent the Pt atoms and the gray circles stand for the Ni atoms. 
Fig 1. G. Wang et al

Fig. 1, G. Wang et al
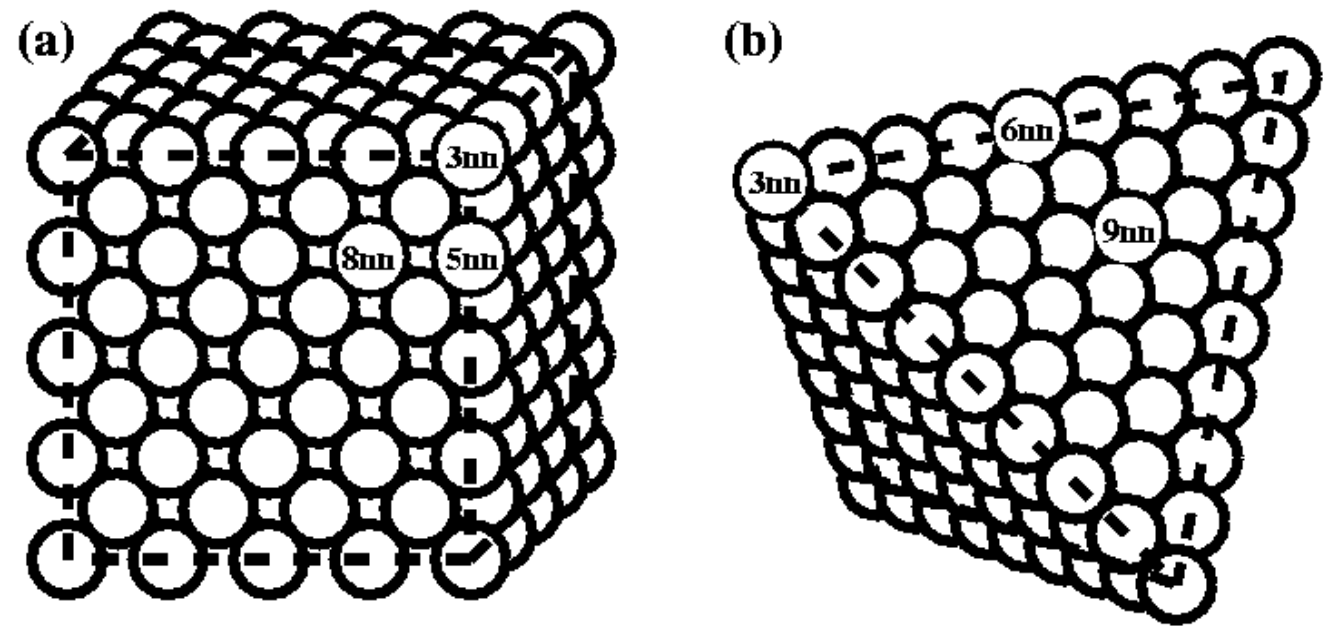

(c)

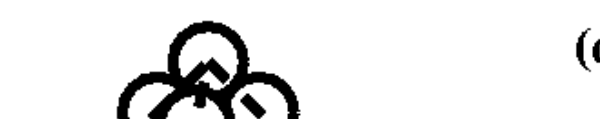

(d)
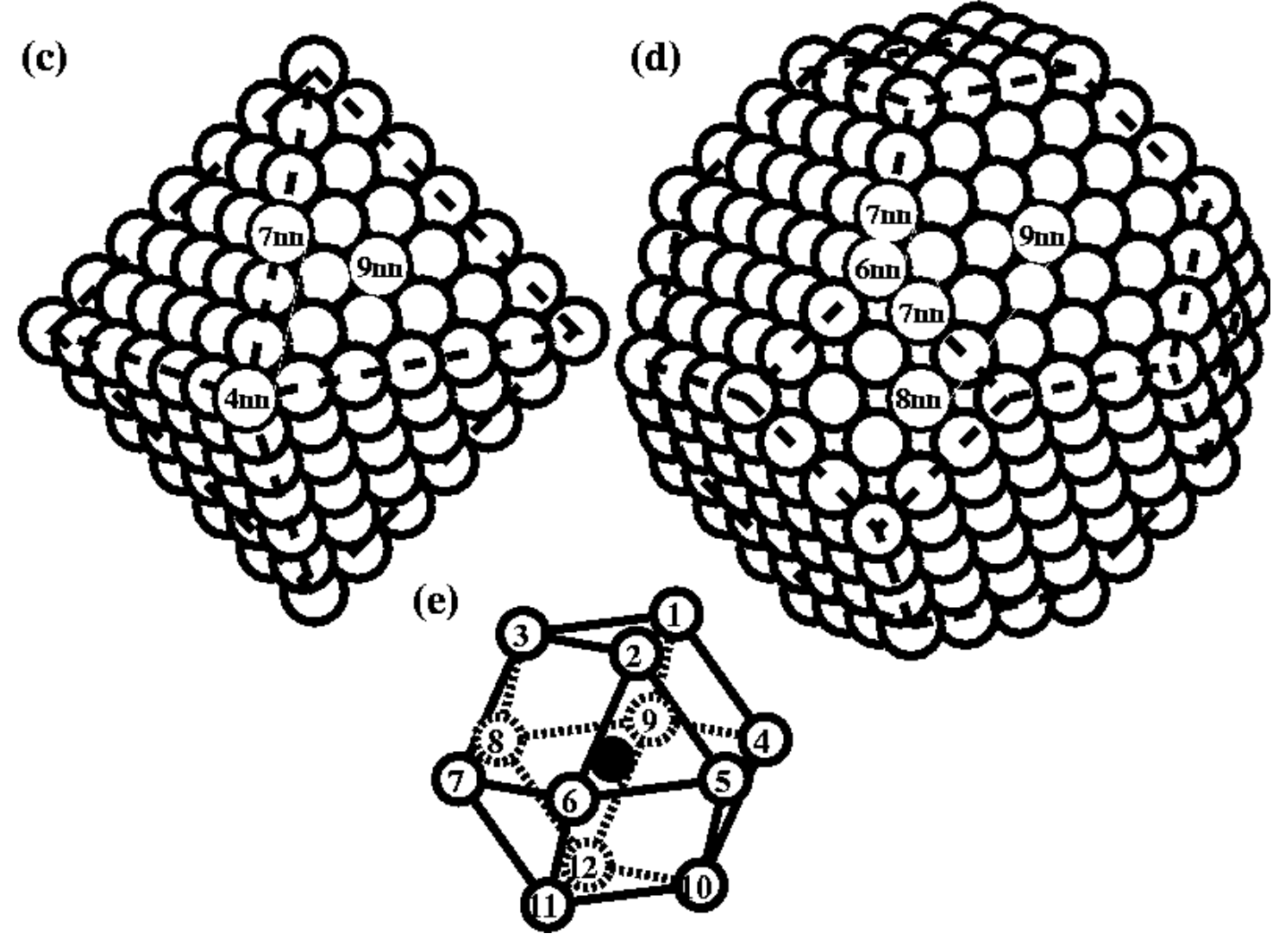
Fig. 2. G. Wang et al

Fig. 2(a) G. Wang et al

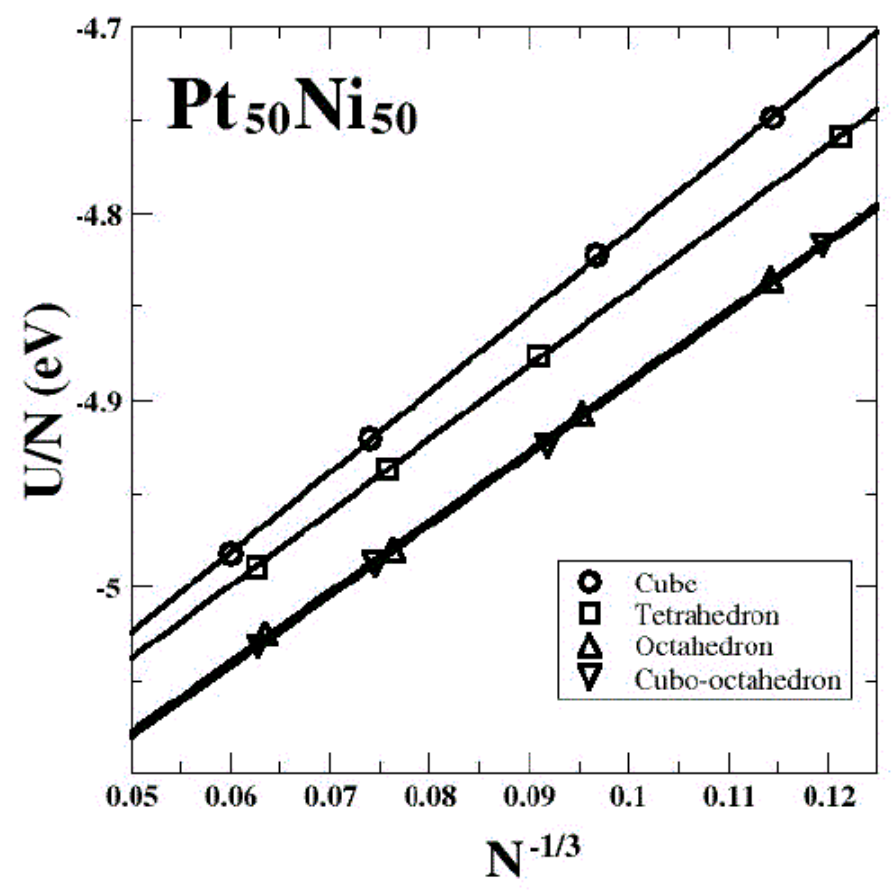

Fig. 2(b) G. Wang et al

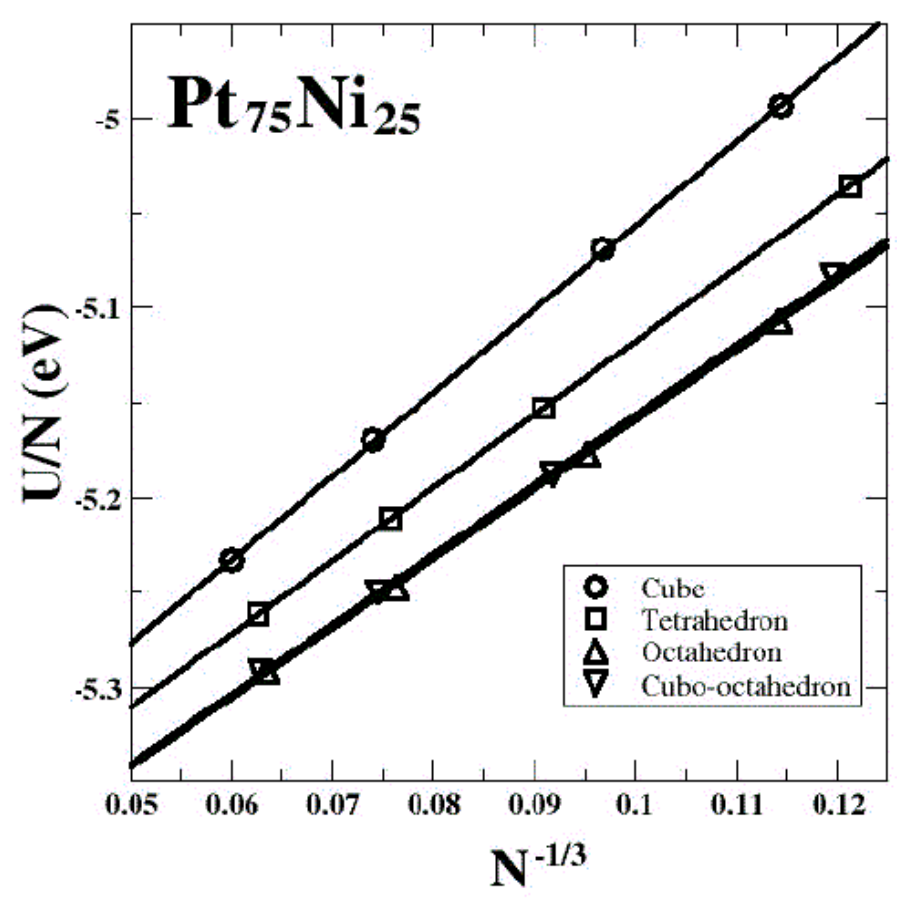


Fig. 3. G. Wang et al

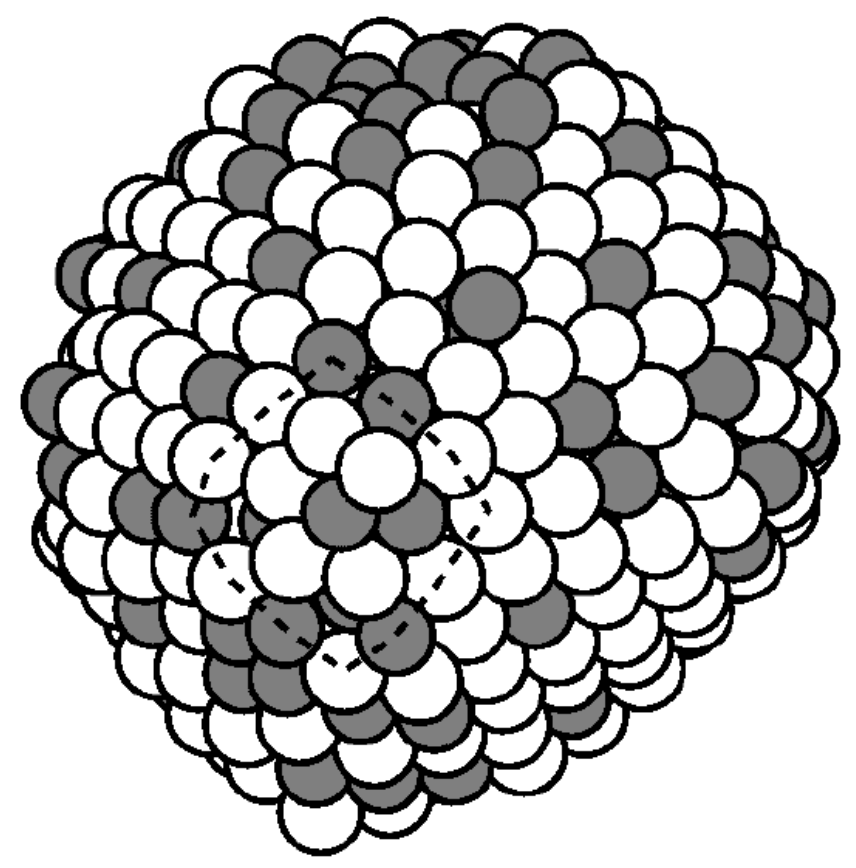

(a) $\mathrm{Pt}_{50} \mathrm{Ni}_{50}$

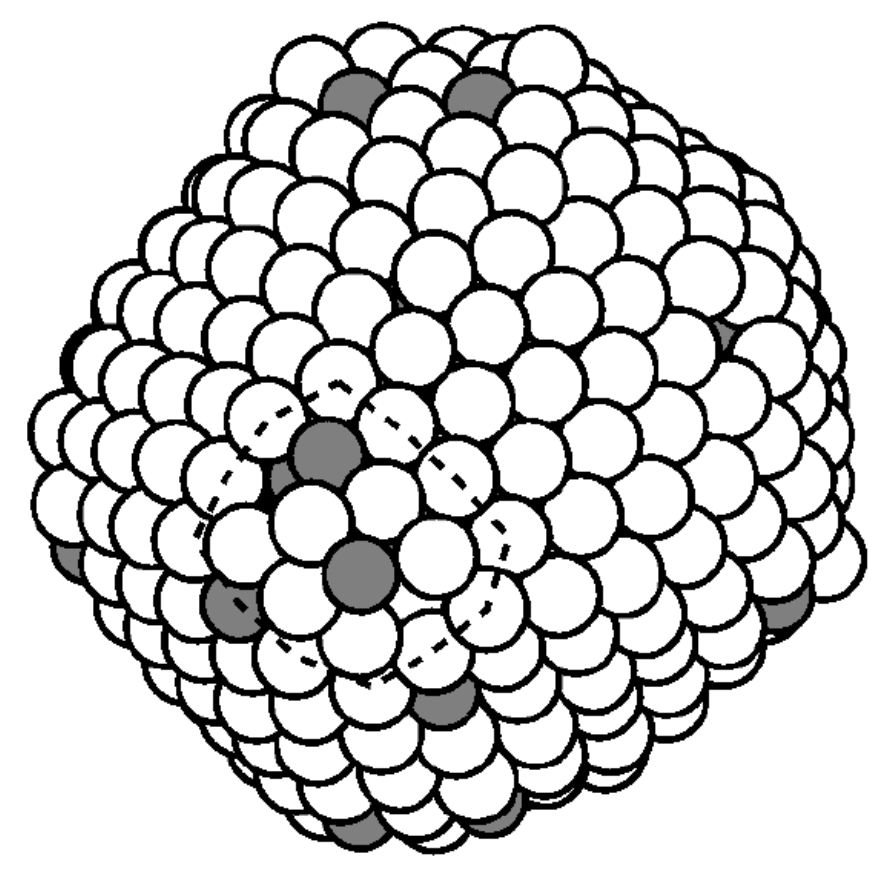

(b) $\mathrm{Pt}_{75} \mathrm{Ni}_{25}$ 
Fig. 4. G. Wang et al

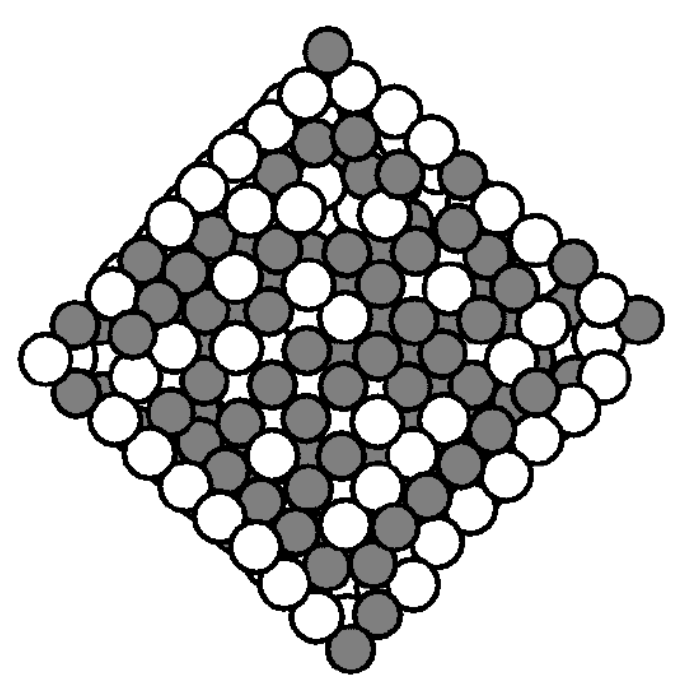

(a) octahedral $\mathrm{Pt}_{50} \mathrm{Ni}_{50}$

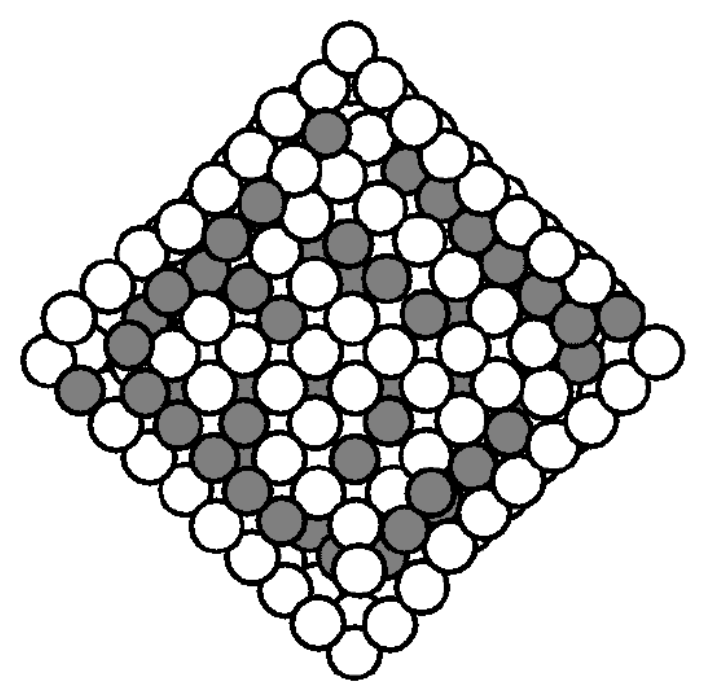

(a) octahedral $\mathrm{Pt}_{75} \mathrm{Ni}_{25}$

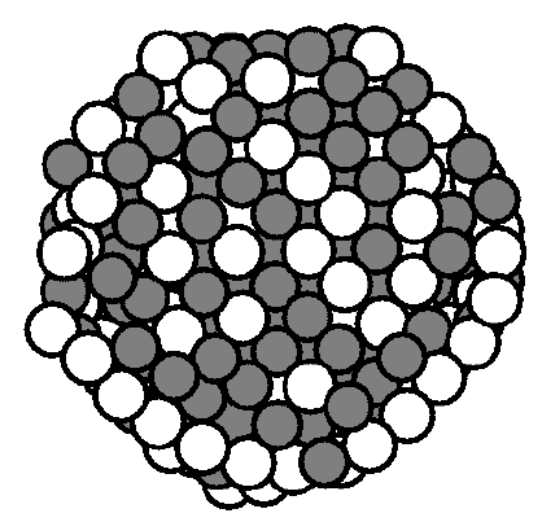

(b) cubo-octadedral $\mathrm{Pt}_{50} \mathrm{Ni}_{50}$

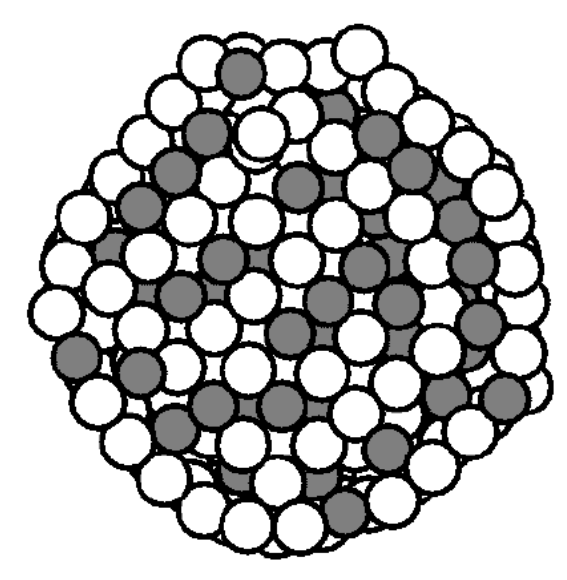

(b) cubo-octadedral $\mathrm{Pt}_{75} \mathrm{Ni}_{25}$ 